\title{
EDITORIAL
}

\section{Blood Lipids: How much low is low enough?}

University Heart Journal 2020; 16(1): 1-2

According to new ESC guidelines of 2019 for the management of dyslipidaemia, there are revised concepts and new recommendations for the management of this condition. Cardiovascular risk categories are based on history of vascular events, coronary and other vascular interventions, documented atherosclerotic cardiovascular disease (ASCVD) on imaging (CAG, CTCA, carotid ultrasound), diabetes (DM) with target organ damage (TOD), CKD, calculated risk score, major risk factors and familial hypercholesterolaemia (FH). Total cardiovascular risk can be estimated by risk scores. ESC recommends the use of SCORE system (Systematic Coronary Risk Estimation) which can be accessed by ( www.HeartScore.org). For risk estimation in Bangladesh WHO risk estimation chart is more appropriate to calculated total cardiovascular risk but ESC risk chart for high-risk countries can be used.

Very high-risk category includes people with documented ASCVD, either clinical or unequivocal on imaging. Documented ASCVD includes previous ACS (MI or unstable angina), stable angina, coronary revascularization (PCI, CABG, and other arterial revascularization procedures), stroke and TIA, and peripheral arterial disease. Unequivocally documented ASCVD on imaging includes those findings that are known to be predictive of clinical events, such as significant plaque on CAG or CTCA (multivessel coronary disease with two major epicardial arteries having $>50 \%$ stenosis), or on carotid ultrasound. DM with target organ damage or at least three major risk factors, or early onset of T1DM of long duration ( $>20$ years). Severe CKD $\left(\mathrm{eGFR}<30 \mathrm{ml} / \mathrm{min} / 1.73 \mathrm{~m}^{2}\right)$. A calculated SCORE $>10 \%$ for 10 -year risk of fatal CVD. FH with ASCVD or with another major risk factor.

For secondary prevention in very-high-risk patients, an LDL-C reduction of $>50 \%$ from baseline and an LDL-C goal of $<1.4 \mathrm{mmol} / \mathrm{L}(<55 \mathrm{mg} / \mathrm{dL})$ are recommended. For patients with ASCVD who experience a second vascular event within 2 years (not necessarily of the same type as the first event) while taking maximally tolerated statin therapy, an LDL-C goal of $<1.0 \mathrm{mmol} / \mathrm{L}(<40 \mathrm{mg} / \mathrm{dL})$ may be considered. In primary prevention, for individuals at very-high risk but without $\mathrm{FH}$, an LDL-C reduction of $>50 \%$ from baseline and an LDL-C goal of $<1.4 \mathrm{mmol} / \mathrm{L}(<55 \mathrm{mg} /$ dL) are recommended. For individuals at very-high risk (that is, with another risk factor but without ASCVD), in primary prevention the same goals for LDL-C lowering should be considered.

High-risk category includes people with: Markedly elevated single risk factors, in particular TC $>8 \mathrm{mmol} / \mathrm{L}$ $(>310 \mathrm{mg} / \mathrm{dL})$, LDL-C $>4.9 \mathrm{mmol} / \mathrm{L}(>190 \mathrm{mg} / \mathrm{dL})$, or BP $>180 / 110 \mathrm{mmHg}$. Patients with FH without other major risk factors. Patients with DM without target organ damage, with DM duration > 10 years or another additional risk factor. Moderate CKD (eGFR 30-59 mL/min/1.73 m²). A calculated SCORE $>5 \%$ and $<10 \%$ for 10 - year risk of fatal CVD.

For patients at high risk, an LDL-C reduction of $>50 \%$ from baseline and an LDL-C goal of $<1.8 \mathrm{mmol} / \mathrm{L}(<70 \mathrm{mg} /$ dL) are recommended.

Moderate-risk category includes young patients (T1DM $<35$ years; T2DM $<50$ years) with DM duration $<10$ years, without other risk factors. Calculated SCORE is between $>1 \%$ and $<5 \%$ for 10 -year risk of fatal CVD.

For individuals at moderate risk, an LDL-C goal of $<2.6$ $\mathrm{mmol} / \mathrm{L}(<100 \mathrm{mg} / \mathrm{dL})$ should be considered.

Low-risk people are those whose calculated SCORE is $<1 \%$ for 10 -year risk of fatal CVD.

For individuals at low risk, an LDL-C goal of $<3.0 \mathrm{mmol} / \mathrm{L}$ $(<116 \mathrm{mg} / \mathrm{dL})$ may be considered.

\section{Which drug or drug combinations are used to achieve cholesterol target?}

Statin remains the mainstay of cholesterol reduction. If the goals are not achieved with the maximum tolerated dose of statin, combination with ezetimibe is recommended. For secondary prevention, patients at very-high risk not achieving their goal on a maximum tolerated dose of statin and ezetimibe, a combination with a PCSK9 inhibitor is recommended. For very-high-risk FH patients (that is, with ASCVD or with another major risk factor) who do not achieve their goals on a maximum tolerated dose of statin and ezetimibe, a combination with a PCSK9 inhibitor is recommended. 


\section{Drug treatments of patients with hypertriglyceridaemia:}

Statin treatment is recommended as the first drug of choice for reducing CVD risk in high-risk individuals with hypertriglyceridaemia [TG $>2.3 \mathrm{mmol} / \mathrm{L}(200 \mathrm{mg} / \mathrm{dL})$.

In high-risk patients with TG between 1.5 and $5.6 \mathrm{mmol} / \mathrm{L}$ (135 - $499 \mathrm{mg} / \mathrm{dL}$ ) despite statin treatment, n-3 PUFAs (icosapent ethyl $2 \times 2 \mathrm{~g}$ /day) should be considered in combination with statins.

In summary, the 2019 ESC Guideline emphasized on CVD risk estimation to decide on initiation of drug treatment for dyslipidaemia apart from life-style modification, ApoB analysis for risk assessment for selected conditions such as high TG level, DM, obesity or very low LDL-C levels and specified treatment with special population such as elderly, DM, ACS, ischaemic stroke, valvular heart disease, $\mathrm{CKD}$ and $\mathrm{PAD}$.

\section{Manzoor Mahmood}

Professor of Cardiology, Bangabandhu Sheikh Mujib Medical University (BSMMU), Dhaka.

\section{Harisul Hoque}

Professor of Cardiology and Head, Division of Heart Failure, Rehabilitation and Preventive cardiology, BSMMU, Dhaka.

\section{Reference:}

12019 ESC/EAS Guidelines for the management of dyslipidaemias: lipid modification to reduce cardiovascular risk accessed online from [https://academic.oup.com/eurheartj/ advance-article/doi/10.1093/eurheartj/ehz455/5556353 on $30 / 10 / 2019]$. 